\title{
Perception paysanne des ligneux à houppier fermé dans les agrosystèmes de Gaya : cas du terroir villageois de Tanda (République du Niger).
}

\author{
Tahirou Sina I ${ }^{1}$, Chaibou $\mathrm{I}^{\star}$, Ngom D ${ }^{3}$, Moussa $\mathrm{H}^{4}$, Banoin $\mathrm{M}^{5}$ \\ 1. Ministère de l'Environnement de la Salubrité et du Développement Durable, BP 578 Niamey (Niger) ; \\ 2. Université Dan Dicko Dankoulodo de Maradi, BP 465 Maradi (Niger); \\ 3 Université Assane Seck de Ziguinchor (Sénégal) ; \\ ${ }^{4}$ Institut National de la Recherche Agronomique du Niger, BP 429 Niamey (Niger) ; \\ ${ }^{5}$ Faculté d'Agronomie, Université Abdou Moumouni, BP 10960 Niamey (Niger). \\ *auteur correspondant : Email : issachaibou@yahoo.fr
}

Original submitted in on $13^{\text {th }}$ November 2015. Published online at www.m.elewa.org on $31^{\text {st }}$ October 2016 http://dx.doi.org/10.4314/jab.v106i1.11

\section{RÉSUMÉ}

Objectifs : Cette étude vise à établir un diagnostic sur la perception paysanne des ligneux à houppier fermé et les pratiques de gestion dans l'agrosystème de Tanda dans la zone nord-soudanienne au Niger

Méthode et résultats : La démarche méthodologique est basée sur une approche systémique prenant en compte la notion d'agrosystème villageois et celle de pratique agroforestière. Ainsi, celles-ci ont été appréhendées à travers des enquêtes auprès de 74 chefs d'exploitation détenteurs d'au moins un champ dans le terroir et des mesures dendrométriques sur les ligneux (hauteur ; diamètre du tronc à $1,3 \mathrm{~m}$; couronne) au niveau de 73 placettes de $2500 \mathrm{~m}^{2}$ chacune. Les résultats montrent que le peuplement ligneux est un parc à Vitellaria paradoxa formé par 31 espèces, reparties en 14 familles, une densité moyenne de 29,25 19,83 individus/hectare et un recouvrement global de $7,87 \%$.

Conclusion et applicabilité des résultats: Dans cet agrosystème, les producteurs distinguent bien les avantages et les inconvénients des espèces ligneuses à houppier fermé. Les espèces qui fournissent des produits forestiers non ligneux à haute valeur marchande comme Vitellaria paradoxa, Adansonia digitata, Parkia biglobosa et Mangifera indica, sont vivement souhaitées et entretenues dans les champs. Par contre, les espèces ligneuses non désirées dans un champ sont les épineuses, celles servant de dortoirs aux oiseaux et celles à effet d'ombrage sur les cultures. Ce qui constitue des paramétres importants pour tous les acteurs des politiques de reboisement dans la zone. Pour palier les effets de ces interactions négatives, les producteurs mettent en œuvre des pratiques agroforestières dont entre autres : l'élagage des ligneux à de moments précis du calendrier cultural, la modification des itinéraires techniques des cultures sous-couvert, la récolte de fourrage pour les animaux en stabulation et la cueillette des produits forestiers non ligneux. Une analyse plus fine des effets de telles pratiques est nécessaire pour élaborer un modèle de fonctionnement plus durable et efficient des systèmes de production.

Mots clés: Agroécologie, Agroforesterie, Système Agraire, Ligneux à Houppier Fermé, Agrosystème, Approche Systèmique, Niger. 

agrosystèmes de Gaya : cas du terroir villageois de Tanda (République du Niger).

\section{ABSTRACT}

Peasant perception of closed crown trees in Gaya agrosystems: Tanda village land (Niger Republic). Objectives: This study aims to establish a diagnosis on the peasant perception of closed crown trees and management practices in the agro-ecosystem of Tanda in the north-Sudanese zone in Niger

Methodology and Results: The methodology was based on a systemic approach taking into account the concepts of agro-ecosystem and agroforestry practice. Thus, they were apprehended through surveys of 74 owners of domain with at least one domain in the locality land and trees measurements (height, diameter at $1.3 \mathrm{~m}$; crown) on 73 plots of $2500 \mathrm{~m}^{2}$ each. The results show that the woody park is formed of 31 species, divided into 14 families, with an average density of $29.25 \pm 19.83$ individuals unit / hectare and an overall recovery of $7.87 \%$.

Conclusions and application of findings: Conclusion and applicability of the results: In this agrosystème, producers distinguish well the advantages and disadvantages of woody species in closed crown. Species that provide non-timber forest products such as high-value Vitellaria paradoxa, Adansonia digitata, Parkia biglobosa and Mangifera indica, are highly desirable and maintained in the field. By cons, unwanted woody plants in a field are thorny, those serving as dormitories for birds and those with shading effect on crops. Which are important parameters for all players reforestation policies in the area. To mitigate the effects of these negative interactions, producers implement agroforestry practices which include: pruning timber at specific times of the crop calendar, changing crop management sub-covered crops, forage harvest animals in stables and gathering of non-timber forest products. A more detailed analysis of the effects of such practices is needed to develop a more sustainable and efficient operating model production.

Key words: Agroecology, Agroforestry, Agrarian system, Practices, Agrosystem, Systemic approach, Niger.

\section{INTRODUCTION}

Le Niger compte 17129076 habitants en 2012 avec un taux d'accroissement annuel moyen de $3,9 \%$ (INS, 2013). Cette forte croissance démographique entraine une forte pression sur les ressources naturelles. Les ligneux constituent la principale ressource énergétique des ménages ruraux, les formations forestières naturelles fournissent environ $87 \%$ des besoins énergétiques des populations (CILSS, 2007). A ce propos, environ 100000 à 120000 hectares de superficies forestières disparaissent chaque année (CNEDD, 2006). L'agriculture praticable sur $12 \%$ de la superficie totale du pays constitue la principale activité socioéconomique de $84 \%$ de la population rurale (INS, 2013) et représente $36,7 \%$ du PIB (Banque Mondiale, 2014). Les rendements agricoles restent faibles du fait de la péjoration climatique et des itinéraires techniques inappropriés (Banoin \& Guengant, 2003). Des investissements publics et privés dans la gestion des ressources naturelles ont été consentis depuis les années 1970 pour améliorer l'efficacité des systèmes de production, mais les résultats sont le plus souvent mitigés (Rochette,
1989 ; Sall et al., 1991 ; Botoni \& Reij, 2009 ; Michiels et al., 2012). Le besoin se fait sentir aujourd'hui de poursuivre les investigations sur les voies et moyens pour réduire les déficits alimentaires, améliorer la fertilité des sols et les rendements des cultures. La pratique de l'agroforesterie ouvre une voie vers la solution au déficit de production agricole, à la pénurie de bois et la dégradation de l'environnement (Kerkhof, 1991 ; Botoni \& Reij, 2009). Cependant, dans les agrosystèmes, les interactions entre les composantes ligneuses et cultures ne sont pas toujours positives (Akpo et al., 2003; Grouzis \& Akpo, 2006 ; Dupraz, 2008). En effet, lorsque les ligneux sont à houppier fermé durant la campagne agricole, les plantes annuelles héliophiles ne se développent pas convenablement sous ces ligneux, car la lumière y constitue l'élément limitant. En conséquence, les espaces couverts par ces espèces ligneuses constituent des terres en moins pour les paysans. Ces types d'arbres sont fréquents dans la zone sahélo-soudanienne et la zone soudanienne qui sont comptées parmi les zones les plus 
Tahirou et al. J. Appl. Biosci. $2016 \quad$ Perception paysanne des ligneux à houppier fermé dans les agrosystèmes de Gaya : cas du terroir villageois de Tanda (République du Niger).

productrices du pays (Banoin \& Guengant, 2003). L'objectif de cette étude est de connaitre la manière dont les producteurs perçoivent et gèrent ces arbres

\section{MATÉRIEL ET MÉTHODE}

Site d'étude : La commune rurale de Tanda $\left(11^{\circ} 56^{\prime}\right.$ et $12^{\circ} 07^{\prime} \mathrm{N}, \quad 03^{\circ} 10^{\prime}$ et $03^{\circ} 25^{\prime} \mathrm{E}$ ) est située dans le département de Gaya au sud-ouest du Niger (figure 1). Elle s'étend sur une superficie d'environ $343 \mathrm{~km}^{2}$ et à houppier fermé dans un agrosystème nord soudanien afin de développer des actions pour améliorer l'efficacité des systèmes de production.

comprend 67 villages administratifs parmi lesquels le terroir de Tanda qui compte 41 hameaux et campements pour une population estimée en 2012 à 8098 habitants (INS, 2014).

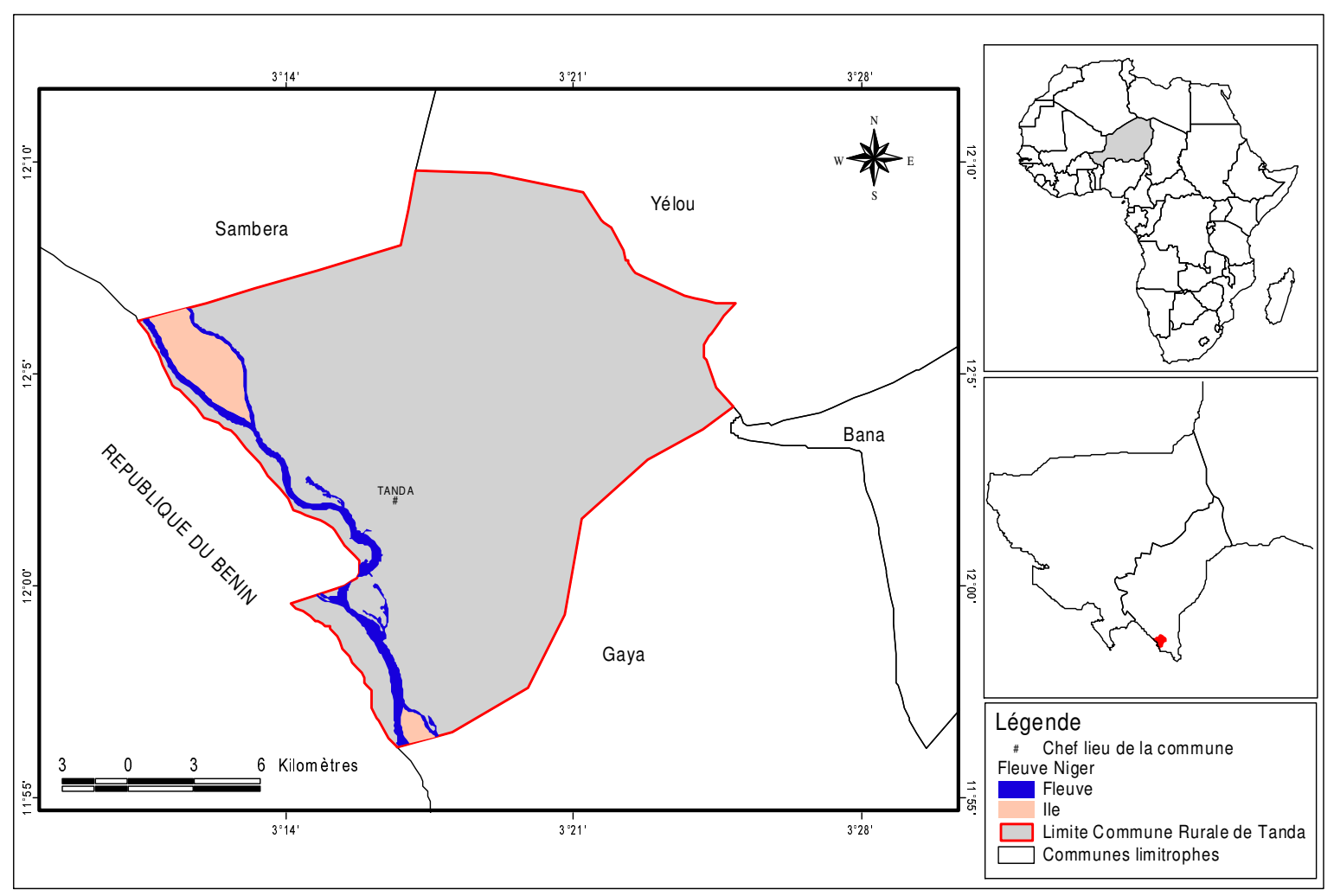

Figure 1 : Localisation de la commune rurale de Tanda (Région de Dosso - sud ouest du Niger)

Le relief est caractérisé par trois grandes unités : le plateau à sommet plat, le glacis et la vallée du fleuve Niger. On distingue 5 types de sols : les sols minéraux bruts d'érosion, les sols peu évolués, les sols ferrugineux tropicaux, les sols ferralitiques et les sols hydromorphes (Dambo, 2001). La moyenne des pluviosités des 15 dernières années à Tanda est de $807 \pm 121 \mathrm{~mm}$. L'évapotranspiration moyenne annuelle s'élève à $2461 \pm 70 \mathrm{~mm}$. Les températures minimale et maximale sont de $18,5^{\circ} \mathrm{C}$ (janvier) et $42^{\circ} \mathrm{C}$ (avril). La circulation atmosphérique est réduite à l'opposition de deux masses d'air : l'harmattan et la mousson. Le climat de Tanda est de type nord soudanien avec une saison de pluies
$(P>2 T)$ de 5 mois et la saison sèche $(P \leq 2 T)$ qui de 7 mois. La période active de la végétation $(P \geq 0,35 x E T P)$ s'étale sur 4 mois (figure 2). Sur le plan phytogéographique, Tanda appartient au compartiment nord-soudanien occidental (Saadou, 1990). Le paysage agraire est dominé par la brousse tigrée sur les plateaux et les parcs agroforestiers sur les terres cultivées (San Emeterio et al., 2013). Les principales activités de la population sont l'agriculture, l'élevage, la pêche, l'apiculture et la commercialisation des fruits forestiers. Le front de mise en culture qui n'était que de $20 \%$ en 1965 atteint 90\% en 2008 (San Emeterio et al., 2013). 

agrosystèmes de Gaya : cas du terroir villageois de Tanda (République du Niger).

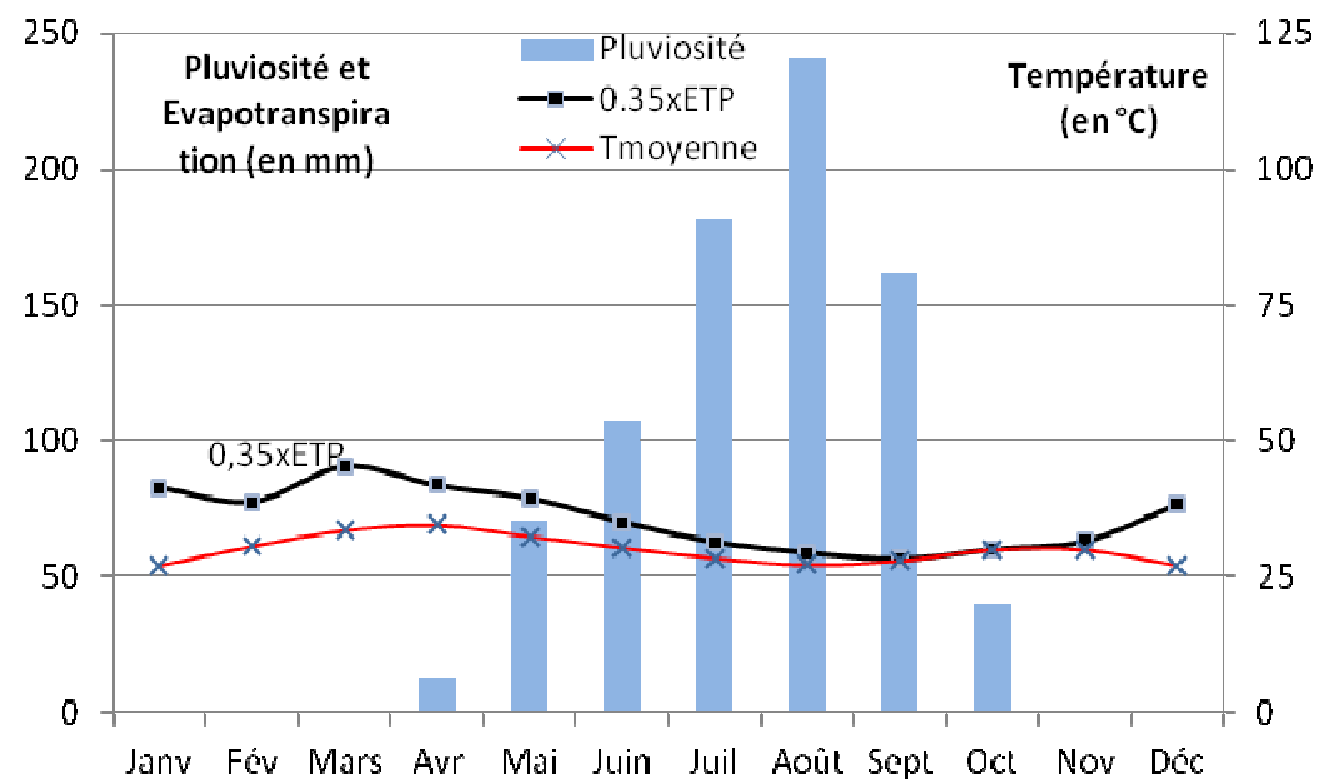

Figure 2 : Diagramme ombrothermique et bilan hydrique à Tanda (Gaya-Dosso)

Collectes et analyse des données: La démarche méthodologique mise en œuvre est basée sur une approche systémique qui a pris en compte la notion d'agrosystème villageois, organisation adoptée par une communauté pour exploiter son espace, gérer ses ressources et subvenir à ses besoins (Jouve \& Tallec, 1994). Une des portes d'entrée pour appréhender les perceptions qu'ont les exploitants des ligneux à houppier fermé réside dans l'analyse des pratiques agroforestières. Une bonne compréhension des pratiques nécessite une analyse croisée de leurs modalités, leurs opportunités et de leurs efficacités (Raison \& Jouve, 1988). Les pratiques agroforestières ont été appréhendées à travers des mesures dendrométriques sur les ligneux et des enquêtes auprès des exploitants détenteurs d'au moins un champ dans un parc agroforestier du terroir villageois.

Mesures dendrométriques sur les ligneux des parcs agroforestiers: Une pré-typologie des parcs agroforestiers de l'agrosystème, basée sur les essences forestières dominantes a été tout d'abord faite. Ensuite, dans chaque type de parc, un échantillonnage systématique a été fait conformément aux recommandations de Mahamane \& Saadou (2008). Finalement, les mesures dendrométriques ont été faites sur 73 placettes de $2500 \mathrm{~m}^{2}$ chacune. Pour chaque placette, un recensement exhaustif de tous les individus ligneux a été fait. Au niveau de chaque individu, les paramètres suivants ont été déterminés.
- le diamètre du tronc a été mesuré à l'aide d'un mètre ruban à $1,30 \mathrm{~m}$ pour les arbres et à $0,3 \mathrm{~m}$ pour les rejets, les arbustes et les arbrisseaux (CTFT, 1989). Les classes de diamètre retenues sont : $[010] \mathrm{cm} ;] 1020] \mathrm{cm} ;] 20$ $30] \mathrm{cm} ;] 3040] \mathrm{cm} ; 14050] \mathrm{cm} ;] 5060]$ et de $60 \mathrm{~cm}$ à plus ;

- la hauteur totale a été déterminée à l'aide des jalons. Elle a permis d'établir la structure verticale du peuplement ligneux. Les strates retenues sont celles des savanes nord soudaniennes. II s'agit notamment de la strate arbustive basse ; [de 0 à $4 \mathrm{~m}[$; la strate arbustive haute [de 0 à $8 \mathrm{~m}[$; la strate arborée basse [de 0 à $16 \mathrm{~m}[$ et la strate arborée haute [de $16 \mathrm{~m}$ et plus[ ;

- le diamètre moyen de la couronne suivant deux axes perpendiculaires, en simulant sa projection verticale au sol. II a servi à déterminer le recouvrement global des ligneux dans les stations.

Enquête auprès des chefs d'exploitation: L'unité d'étude est l'exploitation agricole, car c'est à ce niveau que se prennent les décisions techniques et de gestion relatives aux règles et/ou pratiques agro-sylvo-pastorales (Ouedraogo et al., 2010). Ainsi, l'enquête a été réalisée auprès de 74 chefs d'exploitation soit $10 \%$ des exploitations de Tanda. L'objectif de cette enquête visait à mieux comprendre, face aux contraintes et opportunités de cet agrosystème, les raisons qui poussent l'exploitant à laisser délibérément une espèce ligneuse (plutôt qu'une autre) dans son champ. Elle vise aussi à mieux connaître les espèces ligneuses souhaitées, les avantages et inconvénients liés à la présence des ligneux dans le 

agrosystèmes de Gaya : cas du terroir villageois de Tanda (République du Niger).

champ, les pratiques d'arbitrage des interactions négatives entre les ligneux et leurs systèmes de production.

Traitement et analyse des données: Les données collectées ont été analysées avec le logiciel SPSS. La densité moyenne $(\mathrm{Dm})$ du peuplement ligneux du parc agroforestier a été calculée suivant la formule :

$\mathrm{Dm}=\left(\sum \mathrm{n}_{\mathrm{i}}\right) / \mathrm{N}$, avec $: \boldsymbol{n}_{\boldsymbol{i}}$

le nombre d'individus ligneux par placette et $\boldsymbol{N}$ étant la surface totale des placettes en ha.

\section{RÉSULTATS}

Caractéristiques des parcs à Vitellaria paradoxa: L'inventaire floristique des parcs à Vitellaria paradoxa a permis de dénombrer 31 espèces ligneuses, reparties en 14 familles. Les familles les plus représentées sont les Combretaceae (8 espèces), les Mimosaceae (6 espèces) et les Caesalpiniaceae (4 espèces). Les Anacardiaceae, les Bombacaceae sont représentées chacune par deux espèces et les Arecaceae, les Balanitaceae, les Ebenaceae, les Meliaceae, les Moraceae, les Myrtaceae,
Le recouvrement global $(R)$ a été calculé par la formule : $\mathrm{R}(\mathrm{en} \%)=\sum r_{i} / \mathrm{N}$,

avec: $\mathrm{N}$, le nombre total de placettes, $\boldsymbol{r}_{\boldsymbol{i}}$ est le taux de recouvrement par placette ;

$r_{i}=\sum \Omega(\mathrm{dm} / 2)^{2} / 2500 \mathrm{~m}^{2}(\mathrm{dm}$ est le diamètre moyen de l'individu ligneux sur la placette). Les ligneux souhaités (et non désirés) dans les champs ont été classés en ordre décroissant de citation. L'espèce ayant la fréquence de citation la plus élevée, se trouve attribuer le premier ordre. Puis le second ordre est attribué à l'espèce qui la suit. Ainsi de suite jusqu'à la dernière espèce.

les Sapotaceae, les Sterculiaceae, les Verbenaceae ne sont représentées que par une seule espèce chacune. La densité moyenne est de $29,25 \pm 19,83$ individus/hectare et le recouvrement global des ligneux est de $7,87 \%$. Le peuplement ligneux est dominé par 4 espèces (tableau 1 ) : Vitellaria paradoxa $(20,17 \%)$, Guiera senegalensis $(12,45 \%)$, Piliostigma reticulatum $(7,73 \%)$ et Combretum collinum $(6,44 \%)$.

Tableau 1 : Fréquence et proportion des espèces inventoriées dans le parc agroforestier de Tanda

\begin{tabular}{|l|c|c|c|}
\hline Espèce & Famille & $\begin{array}{c}\text { Nombre } \\
\text { d'individus }\end{array}$ & $\begin{array}{c}\text { Contribution spécifique } \\
\text { (en \%) }\end{array}$ \\
\hline Vitellaria paradoxa & Sapotaceae & 47 & 20,17 \\
\hline Guiera senegalensis & Combretaceae & 29 & 12,45 \\
\hline Piliostigma reticulatum & Caesalpiniaceae & 18 & 7,73 \\
\hline Combretum collinum & Combretaceae & 15 & 6,44 \\
\hline Sclerocarya birrea & Anacardiaceae & 13 & 5,58 \\
\hline Prosopis africana & Mimosaceae & 11 & 4,72 \\
\hline Adansonia digitata & Bombacaceae & 10 & 4,29 \\
\hline Bombax costatum & Bombacaceae & 10 & 4,29 \\
\hline Eucalyptus camaldulensis & Myrtaceae & 9 & 3,86 \\
\hline Lannea microcarpa & Anacardiaceae & 9 & 3,86 \\
\hline Parkia biglobosa & Mimosaceae & 9 & 3,86 \\
\hline Vitex doniana & Verbenaceae & 6 & 2,58 \\
\hline Combretum glutinosum & Combretaceae & 5 & 2,15 \\
\hline Albizia chevalieri & Mimosaceae & 4 & 1,72 \\
\hline Anogeissus leiocarpus & Combretaceae & 4 & 1,72 \\
\hline Combretum micranthum & Combretaceae & 4 & 1,72 \\
\hline Combretum nigricans & Combretaceae & 4 & 1,72 \\
\hline Acacia nilotica & Mimosaceae & 3 & 1,29 \\
\hline Balanites aegyptiaca & Balanitaceae & 3 & 1,29 \\
\hline Tamarindus indica & Cesalpiniaceae & 3 & 1,29 \\
\hline Acacia macrostachya & Mimosaceae & 2 & 0,86 \\
\hline Azadiracta indica & Meliaceae & 2 & 0,86 \\
\hline Combretum fragans & Combretaceae & 2 & 0,86 \\
\hline Diospyros mespiliformis & Ebenaceae & 2 & 0,86 \\
\hline & & & \\
\hline
\end{tabular}


Tahirou et al. J. Appl. Biosci. $2016 \quad$ Perception paysanne des ligneux à houppier fermé dans les agrosystèmes de Gaya : cas du terroir villageois de Tanda (République du Niger).

\begin{tabular}{|l|c|c|c|}
\hline Faidherbia albida & Mimosaceae & 2 & 0,86 \\
\hline Sterculia setigera & Sterculiaceae & 2 & 0,86 \\
\hline Borassus aethiopum & Arecaceae & 1 & 0,43 \\
\hline Cassia sieberiana & Caesalpiniaceae & 1 & 0,43 \\
\hline Daniellia oliveri & Caessalpiniaceae & 1 & 0,43 \\
\hline Ficus ingens & Moraceae & 1 & 0,43 \\
\hline Terminalia avicennioides & Combretaceae & 1 & 0,43 \\
\hline Total 31 & $\mathbf{1 4}$ & $\mathbf{2 3 3}$ & $\mathbf{1 0 0 , 0 0}$ \\
\hline
\end{tabular}

L'analyse de la distribution verticale des ligneux montre que toutes les classes de hauteur définies sont relativement bien représentées (figure 3). La classe la plus rencontrée est celle de 8 à 16 m avec une proportion de $40,77 \%$, puis viennent respectivement les classes de 0 à $4 \mathrm{~m}(31,33 \%)$, de 4 à $8 \mathrm{~m}(20,17 \%)$ et celle de $16 \mathrm{~m}$ à plus $(7,73 \%)$.

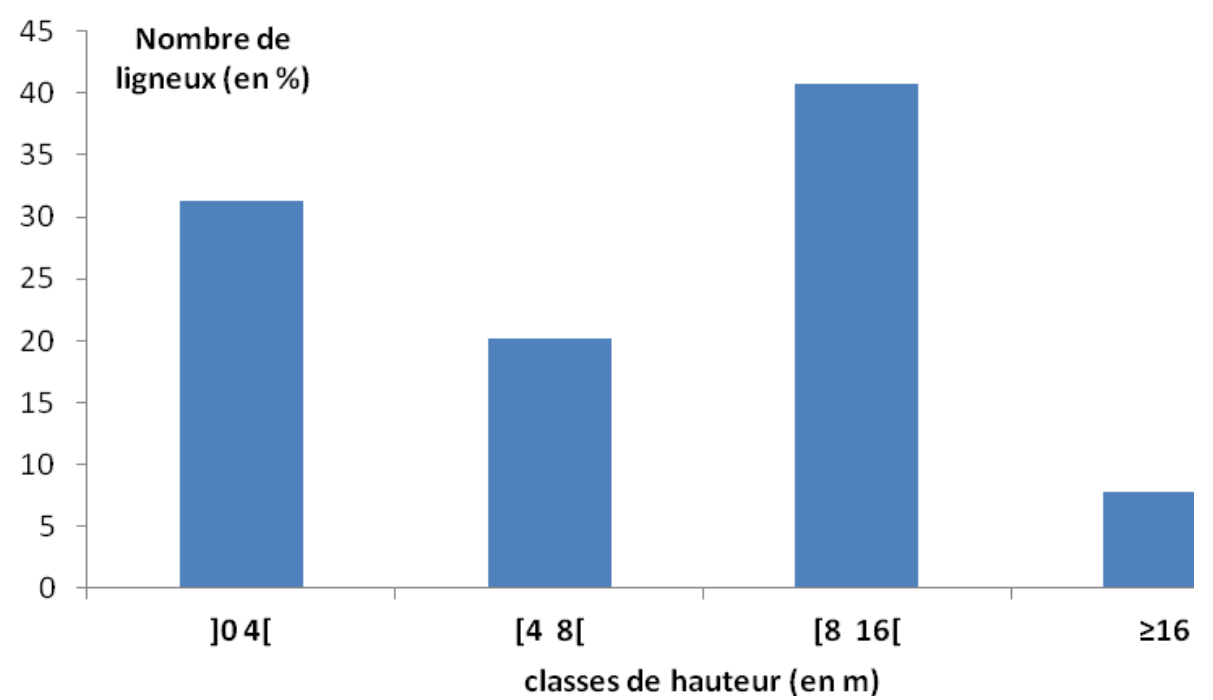

Figure 3 : Distribution des ligneux du parc agroforestier par classe de hauteur

La distribution des ligneux par classe de diamètre (figure 4) montre aussi que toutes les classes sont bien représentées. La classe la mieux représentée est celle de
0 à $10 \mathrm{~cm}$ avec une proportion de $27,9 \%$. Ce qui dénote que le parc à Vitellaria paradoxa de cet agrosystème est en construction. 

agrosystèmes de Gaya : cas du terroir villageois de Tanda (République du Niger).

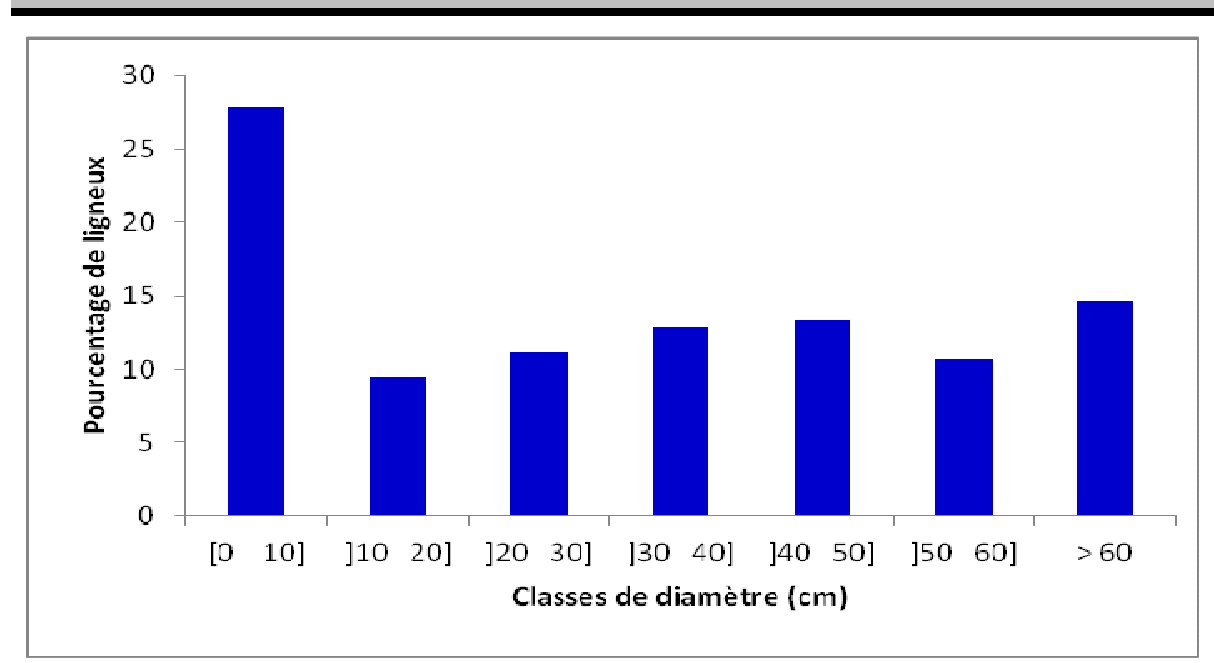

Figure 4 : Distribution des ligneux du parc agroforestier par classe de diamètre

Espèces ligneuses souhaitées dans l'agrosystème de Tanda: Les espèces ligneuses les plus vivement souhaitées sont entre autres selon leur ordre de préférence : Vitellaria paradoxa Gaertn.F. ; Adansonia digitata L. ; Mangifera indica L. ; Citrus sp; Psidium guajava L. ; Parkia biglobosa (Jacq) R. Br. Ex G. Don ; Borassus aethiopum Mart. (Tableau 2). La préférence accordée à ces espèces est surtout liée à leur usage alimentaire. Les espèces ligneuses non désirées dans les champs sont surtout celles à houppier fermé et étendu qui créent de l'ombre aux cultures et celles qui ne produisent aucun organe comestible. Puis viennent les espèces épineuses, celles servant de dortoir aux oiseaux et celles objet de coupes par les bergers transhumants.

Tableau 2 : Espèces ligneuses souhaitées ou non dans les champs de l'agrosystème de Tanda (Dosso)

\begin{tabular}{|c|c|c|c|c|c|c|c|c|c|c|}
\hline \multirow[t]{2}{*}{ Espèces } & \multicolumn{5}{|c|}{$\begin{array}{l}\text { Espèces ligneuses souhaitées et } \\
\text { raisons avancées }\end{array}$} & \multicolumn{5}{|c|}{$\begin{array}{l}\text { Espèces ligneuses non souhaitées et } \\
\text { raisons invoquées }\end{array}$} \\
\hline & RANG & VM & FOU & PHAM & BOS & RANG & EPI & EOM & DORT & APC \\
\hline Vitellaria paradoxa & 1 & $x$ & & & & 18 & & $x$ & & \\
\hline Adansonia digitata & 2 & $x$ & & & & 19 & & $x$ & & \\
\hline Mangifera indica & 3 & $\mathrm{x}$ & & & & & & & & \\
\hline Parkia biglobosa & 4 & $x$ & & & & 16 & & $\mathrm{x}$ & & \\
\hline Borassus aethiopum & 5 & $x$ & & & & & & & & \\
\hline Anacardium occidentale & 6 & $x$ & & & & & & & & \\
\hline Tamarindus indica & 7 & $x$ & & & & 16 & & $x$ & & \\
\hline Vitex doniana & 7 & $x$ & & & & & & & & \\
\hline Lannea microcarpa & 8 & $x$ & & & & 17 & & $x$ & & \\
\hline Sclerocarya birrea & 9 & $x$ & & $x$ & $x$ & 13 & & $x$ & & \\
\hline Prosopis africana & 10 & & & & $x$ & 16 & & $x$ & & $x$ \\
\hline Maerua oblongifolia & 11 & & & $\mathrm{x}$ & & & & & & \\
\hline Khaya senegalensis & 13 & & & & $x$ & & & & & \\
\hline Cordyla pinnata & 14 & & $x$ & & & & & & & \\
\hline Cassia sieberiana & 15 & & & $x$ & & & & $x$ & & $x$ \\
\hline Balanites aegyptiaca & & & & & & 1 & $x$ & $x$ & & \\
\hline Bombax costatum & & & & & & 2 & $\mathrm{x}$ & & & \\
\hline Albizia chevalieri & & & & & & 3 & & & & $x$ \\
\hline Acacia ataxancantha & & & & & & 4 & $x$ & & & \\
\hline
\end{tabular}


Tahirou et al. J. Appl. Biosci. $2016 \quad$ Perception paysanne des ligneux à houppier fermé dans les agrosystèmes de Gaya : cas du terroir villageois de Tanda (République du Niger).

\begin{tabular}{|c|c|c|c|c|c|c|c|c|c|}
\hline Acacia senegal & & & & & 5 & $x$ & & & \\
\hline Faidherbia albida & & & & & 5 & $x$ & & $x$ & \\
\hline Albizia malacophylla & & & & & 5 & $x$ & & $x$ & \\
\hline Acacia nilotica & & & & & 6 & $x$ & $x$ & & \\
\hline Acacia polyacantha & & & & & 7 & $x$ & & & $x$ \\
\hline Ziziphus mauritana & & & & & 8 & $x$ & & & \\
\hline Sterculia setigera & & & & & 9 & & $x$ & & $x$ \\
\hline Anogeissus leiocarpus & & & & & 9 & & $x$ & & $x$ \\
\hline Piliostigma reticulatum & & & & & 10 & & $x$ & & \\
\hline Mitragyna inermis & & & & & 13 & & $X$ & & $x$ \\
\hline Ficus ingens & & & & & 14 & & $x$ & & \\
\hline Combretum glutinosum & & & & & 15 & & $x$ & & $x$ \\
\hline TOTAL & 10 & 1 & 3 & 3 & & 9 & 16 & 2 & 8 \\
\hline
\end{tabular}

Légende

VM : Production non ligneuse à haute valeur marchande

FOU : Espèce fourragère

PHAM : Produit pharmaco-magique

BOS : Bois d'œuvre et de service

Arbre à houppier fermé et système de culture : Dans cet agrosystème nord-soudanien, les producteurs distinguent 3 avantages majeurs des ligneux à houppiers fermés sur les systèmes de cultures: protection des cultures contre la verse (93\% des personnes enquêtées), apport en matières organiques au sol (49\%) et création d'un microclimat favorable (41\%). Les principaux inconvénients ou interactions négatives liées leurs présence dans les cultures signalés par les exploitants sont : dégâts des oiseaux (85\%), effet ombrage (54\%) et chute des branches sur les cultures (22\%). Pour atténuer les effets de ces interactions négatives, les producteurs élaguent les branches lors de la préparation des champs et modifient certaines pratiques culturales sous couvert des ligneux à houppiers fermés. En ce qui concerne la coupe des branches, elle est faite régulièrement par $76 \%$ des paysans. Les branches qui font l'objet de coupes fréquentes sont les branches pendantes (43\%), puis les branches basales (41\%) et enfin les branches buissonnantes (34\%). La principale raison profonde pour laquelle $65 \%$ des exploitants pratiquent cet élagage est de favoriser la pénétration de la lumière sous les arbres au profit des cultures qui s'y trouvent. Mais $11 \%$ des exploitants affirment se procurer du bois à travers cette pratique. Aussi, les branches coupées contribuent à l'alimentation du bétail, surtout en saison sèche. Les exploitants qui ne pratiquent pas des coupes à chaque campagne agricole (24\%) s'abstiennent par crainte de représailles de la part des agents du service des eaux et forêts. A propos de l'espace sous-couvert ligneux, il est
EPI : Espèce épineuse

EOM : Espèce à effet d'ombre

DORT: dortoir d'oiseaux

APC : aucune production comestible

géré de deux manières : $84 \%$ sèment à des densités de semis similaires $(64 \%)$ ou inférieures $(20 \%)$ à celles de l'espace hors-couvert et $16 \%$ des producteurs n'y sèment rien. L'objectif visé par ces pratiques est double. Les uns visent à optimiser les rendements des cultures $(41 \%)$ et les autres visent à pouvoir désherber l'espace souscouvert pour servir d'abris pour le repos lors des travaux champêtres (60\%).

Arbre à houppier fermé et système d'élevage : La population de ce terroir agricole est unanime que les ligneux à houppier fermé dans les champs deviennent de plus en plus des pôles d'attraction des animaux au pâturage surtout en saison sèche chaude en quête de fourrage aérien ou d'un abri pour se protéger des températures élevées. $31 \%$ des enquêtés affirment récolter les feuilles de ces ligneux pour constituer des réserves stratégiques de fourrages pour leurs animaux en stabulation. Mais l'inconvénient majeur qui limite l'entretien de ces ligneux dans les champs, c'est qu'ils font l'objet de coupes répétitives par les bergers transhumants.

Arbre à houppier fermé et autres activités socioéconomiques: Dans l'agrosystème de Tanda, les activités ayant un rapport avec les ligneux à houppier fermé sont l'apiculture et la cueillette des produits forestiers à laquelle sont affiliées la transformation et la commercialisation des fruits des ligneux à houppier fermé. II ressort de l'enquête que $96 \%$ des exploitants s'y procurent des produits alimentaires ; $89,19 \%$ font des prélèvements de produits de pharmacopée et $39,19 \%$ 


\section{Tahirou et al. J. Appl. Biosci. $2016 \quad$ Perception paysanne des ligneux à houppier fermé dans les}

agrosystèmes de Gaya : cas du terroir villageois de Tanda (République du Niger).

des chefs d'exploitation pratiquent l'apiculture dans le parc agroforestier. Quant aux bois énergie, de service ou d'œuvre, toutes les familles s'en procurent quand l'opportunité se présente. $58,11 \%$ des chefs d'exploitation enquêtés commercialisent les produits forestiers non ligneux provenant du parc agroforestier et obtiennent des précieux revenus (tableau 3).

Tableau 3 : Principaux produits forestiers de cueillette et leurs valeurs marchandes locales

\begin{tabular}{|l|l|l|}
\hline Espèces et produits forestiers & $\begin{array}{l}\text { Conditionnement (sac } \\
\text { de } 100 \mathrm{~kg} \text { ) }\end{array}$ & $\begin{array}{l}\text { Coût au producteur } \\
\text { selon les fluctuations du } \\
\text { marché (Fcfa) }\end{array}$ \\
\hline Vitellaria paradoxa Gaertn. f. (fruits de karité) & sac de $100 \mathrm{~kg}$ & 4000 à 6000 \\
\hline Vitellaria paradoxa Gaertn. f. (noix de karité) & sac de $100 \mathrm{~kg}$ & 2500 à 10000 \\
\hline Parkia biglobosa (Jacq)R.Br. ex G.Don (gousses du néré) & sac de $100 \mathrm{~kg}$ & 3000 à 10000 \\
\hline Adansonia digitata L. (feuilles séchées de baobab) & sac de $100 \mathrm{~kg}$ & 1000 à 2500 \\
\hline Tamarindus indica L. (gousses du tamarinier) & sac de $100 \mathrm{~kg}$ & 7500 à 10000 \\
\hline Miel & Litre & 500 à 2500 \\
\hline
\end{tabular}

\section{DISCUSSION}

Caractéristiques des parcs à Vitellaria paradoxa : Le nombre d'espèces ligneuses inventoriées dans cet agrosystème nord soudanien (31 espèces) ainsi que leur densité $(29,25 \pm 19,83$ individus par hectare) et leur recouvrement $(7,87 \%)$ sont assez faibles. Cette richesse spécifique du parc agroforestier est proche de celle du parc agroforestier à Vitellaria paradoxa du finage de Boumba (Gaya - Niger) où 35 espèces ont été inventoriées (Dan Guimbo et al., 2010). Par contre, dans les parcs à Sclerocarya birrea du complexe des forêts classées Dan Kada de la région de Maradi (Niger), seulement 20 espèces ligneuses ont été obtenues (Abdourhamane et al., 2013). Au Burkina Faso, dans le domaine nord-soudanien (pluviosité annuelle de 761 $\mathrm{mm}$ ), il a été rapporté un nombre plus élevé d'espèces ligneuses (41 espèces) dans les parcs à Vitellaria paradoxa du terroir de Vipalogo (Yaméogo et al., 2005). La caractérisation des parcs agroforestiers du terroir de Mar Fafaco (Fatick-Sénégal) a aussi révélé un meilleur effectif d'espèces ligneuses rencontrées : 54 espèces (Diedhou et al., 2014). En général, le nombre réduit d'espèces ligneuses observées dans les parcs agroforestiers, serait lié à la forte sélection et choix des espèces végétales qui seront conservées, entretenues ou coupées lors de la préparation des champs (Devineau, 2012 ; San Emeterio et al., 2013 ; Yelemou et al., 2013). Le recouvrement ligneux du parc à Vitellaria paradoxa de Tanda $(7,87 \%)$ est meilleur à celui du complexe Dan Kada qui n'est que de 5,55\% (Abdourhamane et al., 2013), mais, il est inférieur à ceux obtenus dans les parcs à Neocarya macrophylla des autres agrosystèmes de Gaya et de Birni N'Gaouré - 17\% (Dan Guimbo et al., 2010). Ce faible taux de recouvrement à Tanda peut s'expliquer par l'intensité des pratiques d'exploitation des ligneux par la population locale pour le maintenir en deçà de $60 \%$, seuil à partir duquel l'effet bénéfique de l'arbre s'annule (Grouzis \& Akpo, 2006). Ce taux de recouvrement observé dans cet agrosystème permet aussi d'assurer l'éclairement des cultures à un niveau supérieur à $70 \%$, puisque si celui-ci tombe en dessous, le rendement diminue vite (Dupraz, 2008).

Arbre à houppier fermé et système de production : Concernant les espèces ligneuses préférées dans les champs, on remarque que seule la valeur marchande des produits que peuvent fournir les essences forestières intéresse les paysans. En effet, les produits de la phytodiversité des systèmes des parcs agroforestiers constituent une source monétaire pour les ménages qui les récoltent. Ces produits sont demandés sur tous les marchés locaux et souvent exportés vers les pays voisins. Selon les paysans, avoir des espèces ligneuses productrices de produits à valeur marchande dans son champ équivaut à un capital bien placé, car la cueillette de ces produits génère des revenus tout au long de l'année et leurs entretiens ne requièrent que peu ou prou d'investissement. Plusieurs études menées dans diverses zones agro-écologiques montrent que le défrichement est sélectif et les espèces conservées ou entretenues dans les champs sont fonction des conditions écologiques, des savoirs et des besoins des populations et de leur environnement socio-économique (Smektala et al., 2005 ; Yaméogo et al., 2005; Yélémou et al., 2010; Gomgnimbou et al., 2010). Les espèces ligneuses non désirées dans les champs sont surtout celles à houppier fermé et étendu qui créent de l'ombre aux cultures empêchant celles-ci d'exploiter de manière optimale leurs 
effets positifs sur la disponibilité en eau et en nutriments (Breman \& Kessler, 1995). De plus, des espèces comme Acacia nilotica, en plus de l'effet ombrage, ont un effet dépressif sur le rendement des cultures (Emtehani \& Tabari, 2007). Les espèces épineuses gênent les paysans pendant les travaux champêtres. C'est pour atténuer ces interactions négatives que les agriculteurs

\section{CONCLUSION}

On retiendra de cette étude que certes plusieurs facteurs déterminent la présence de l'arbre à houppier fermé dans l'agrosystème nord-soudanien de Tanda. Mais, c'est surtout la valeur marchande des produits que peut fournir une espèce ligneuse qui retient l'attention d'un producteur. Ainsi, ces facteurs doivent être pris en compte par les acteurs intervenant dans le reboisement et la gestion des écosystèmes de la zone nordsoudanien. Pour essayer d'atténuer les effets négatifs liés à la présence de ces espèces, le producteur met en œuvre un certain nombre de pratiques agroforestières comme l'élagage des ligneux et des itinéraires techniques singuliers spécifiques àl'espace sous-couvert ligneux. En termes de perspective, il est nécessaire de poursuivre des investigations pour élaborer des modèles en vue d'optimiser la productivité des parcs agrofrestiers dans

\section{RÉFÉRENCES BIBLIOGRAPHIQUES}

Abdourhamane $\mathrm{H}$, Morou B, Rabiou H, Mahamane A, 2013. Caractéristiques floristiques, diversité et structure de la végétation ligneuse dans le Centre-Sud du Niger : cas du complexe des forêts classées de Dan kada Dodo-Dan Gado. Int. J. Biol. Chem. Sci. 7 : 1048-1068.

Akpo LE, Banoin M, Grouzis M, 2003. Effet de l'arbre sur la production et la qualité fourragères de la végétation herbacée : bilan pastoral en milieu sahélien. Revue Elev. Méd. vét. Pays trop 154 : 619-628.

Banoin, $M$ et Guengant JP, 2003. Dynamique des populations, disponibilités en terres et adaptation des régimes fonciers: le cas du Niger. Gendreau F. Lututala M.(Editor), FAO, Rome, Italie, $144 \mathrm{pp}$.

Banque Mondiale, 2014. Base de données en ligne, www.donnees.banquemondiale.org/indicateur $/ \mathrm{N}$ V.AGR.TOTL.ZS

Botoni $E$ et Reij C, 2009. La transformation silencieuse de l'environnement et des systèmes de production au Sahel : Impacts des investissements publics et privés dans la gestion des ressources naturelles, Centre for procèdent régulièrement à leur élagage. II faut donc comprendre l'élagage opéré par les cultivateurs comme une pratique agricole visant l'amélioration de l'efficience des systèmes de production et non une intention de destruction de la nature comme le supposent souvent les services de l'environnement.

différents systèmes agraires à travers la détermination d'une valeur minimale du rapport: Hauteur de tronc I Diamètre de la couronne, d'un ligneux à houppier fermé, pour assurer l'accès optimum de la lumière diffuse et latérale sous le feuillage permettant un bon développement des principales spéculations. En outre, certes, le bénéfice économique résultant de l'exploitation des produits des arbres est plus élevé que les pertes des rendements des cultures céréalières poussant à l'ombre des ligneux implantés dans les champs. Mais, il est important d'élaborer un modèle d'élagage des arbres qui permettrait de minimiser les pertes de rendement des cultures sous l'ombre des ligneux tout en sécurisant les bénéfices économiques provenant de l'exploitation des produits des arbres.

International Cooperation (CIS), Comité permanent Inter- États de Lutte contre la Sécheresse dans le Sahel (CILSS), 63 pages. www.cilss.bf/IMG/pdf/Rapport_Synthese_Etude SSahel_Final.pdf

Breman H et Kessler JJ, 1995. Le rôle des ligneux dans les agrosystèmes des régions semi-arides (avec un accent particulier sur les pays sahéliens). www.beep.ird.fr/collect/upb/index/assoc/.../IDR2002-SAV-PAT. $149 \mathrm{p}$.

CILSS, 2007. Programme Régional de promotion des Energies Domestiques Alternatives au Sahel (PREDAS), Expérience du Niger en matière de mise en œuvre du Mécanisme pour un développement Propre (MDP). $36 \mathrm{pp}$. www.agrhymet.ne/portailCC/images/pdf/Experie nce\%20MDP_Niger.pdf

CNEDD, 2006. Programme d'Action National pour I'Adaptation aux changements climatiques. www.cnedd.ne/doc_cnedd/PANA_RESILLIENC E/PANA-NIGER.pdf.82p.

CTFT, 1989. Mémento du forestier « techniques rurales en Afrique ». 3ème édition, Paris, France.1266 pp. 
Dambo L, 2001. Atlas de l'arrondissement de Gaya. Mémoire de Maîtrise, Université Abdou Moumouni, Niamey, Niger. 105 p.

Dan Guimbo I, Mahamane A, Ambouta KJM, 2010. Peuplement des parcs à Neocarya macrophylla (Sabine) Prance et à Vitellaria paradoxa (Gaertn. C.F.) dans le sud-ouest nigérien : diversité, structure et régénération. Int. J. Biol. Chem. Sci. 4 : 1706-1720.

Devineau JL, 2012. Écologie des principales espèces ligneuses alimentaires et fourragères dans un système culture-jachère (sud-ouest du Burkina Faso). Floret Ch \& Pontanier R (éditeurs) John Libbey Eurotext, Paris, France, $1:$ 441-450.

Diedhou MAA, Faye E, Ngom D, Touré MA, 2014. Identification et caractérisation floristiques des parcs agroforestiers du terroir insulaire de Mar Fafaco (Fatick, Sénégal). Journal of Applied Biosciences $79: 6855-6866$.

Dupraz C, 2008. Arbres et cultures : l'affaire rebondit en chine! Agroforesteries la revue française des arbres ruraux 1:11-15.

Emtehani MH et Tabari M, 2007. Acacia nilotica and Medicago sativa, suitable plants for agro-forestry in southern coasts of Iran. Pakistan Journal of Biological Sciences 10 : 1713-1717.

Gomgnimbou APK, Savadogo PW, Nianogo AJ, MillogoRasolodimby J, 2010. Pratiques agricoles et perceptions paysannes des impacts environnementaux de la cotonculture dans la province de la Kompienga (Burkina Faso). Sciences \& Nature $7: 165-175$.

Grouzis M et Akpo LE, 2006. Interactions arbre-herbe au Sahel. Sécheresse $17:$ 318-325.

INS, 2013. Résultats du quatrième Recensement Général de la Population et de l'Habitat (RGP/H, 2012), Ministère des Finances, Niamey, Niger. 10 p www.stat-niger .org/statistique/file/rgph2012.pdf.

INS, 2014. Répertoire National des localités (ReNaLoc), Niamey, Niger, Edition juillet 2014, $718 \mathrm{p}$.

Jouve $P$ et Tallec M, 1994. Une méthode d'étude de systèmes agraires en Afrique de l'Ouest par l'analyse de la diversité et de la dynamique des agrosystèmes villageois. Les Cahiers de la Recherche Développement 39 : 43-59.

Kerkhof $P, 1991$. L'agroforesterie en Afrique, l'harmattanInstitut Panos, Paris, France. $254 \mathrm{p}$.

Mahamane A et Saadou M, 2008. Méthode d'étude et d'analyse de la flore et de la végétation tropicale. Acte de l'atelier sur l'harmonisation des méthodes, tenu à Niamey du 4 au 9 Août 2008.

Michiels D, Egg J, Blein R, 2012. La répétition des crises alimentaires et nutritionnelles au Niger : la rénovation urgente des politiques de sécurité alimentaire. Cah. Agric. 21 : 302-310.

Ouédraogo M, Dembélé Y, Somé L, 2010. Perceptions et stratégies d'adaptation aux changements des précipitations : cas des paysans du Burkina Faso. Sécheresse $21: 87-96$.

Raison JP et Jouve P, 1988. Les parcs en Afrique : états des connaissances, perspectives de recherches. Centres d'Etudes Africaines, EHESS, Paris, France. www.Fao.org/DOCREP/005/X3940E/ X3940E04.htm

Rochette RM, 1989. Le Sahel en lutte contre la désertification, leçon d'expérience, Margraf, weikerheim (All.), Cilss - Pac - G.T.Z. 592 pp.

Saadou $M, 1990$. La végétation des milieux drainés nigériens à l'est du fleuve Niger, thèse d'état, Université de Niamey, Niger. 395 pp.

Sall PN, Aussenac G, Dreyer E, Garnier A, 1991. Limitation par la sécheresse de la croissance d'Eucalyptus camaldulensis Dehn. En climat sahélo-soudanien au Sénégal. Rev For Fr XLIII : 309-316.

San Emeterio JL, Alexandre F, Andrieu J, Génin A, Mering C, 2013. Changements socioenvironnementaux et dynamiques des paysages ruraux le long du gradient bioclimatique nordsud dans le sud-ouest du Niger (régions de Tillabery et de Dosso). Vertigo, la revue électronique en science de l'environnement 13. www.vertigo.revues.org/14456

Smektala G, Peltier R, Sibelet N, Leroy M, Manlay R, Njiti CF, Ntoupka M, Njiemoun A, Palou O, Tapsou, 2005. Parcs agroforestiers sahéliens: de la conservation à l'aménagement. Vertigo, la revue électronique en science de l'environnement 6. www. vertigo.revues.org/4410

Yameogo G, Yelemou B, Traoré D, 2005. Pratique et perception paysannes dans la création de parc agroforestier dans le terroir de Vipalogo (Burkina Faso). Base 9 : 241-248www.popups.ulg.ac.be/ 1780-4507/index.php?id=1404.

Yélémou B, Yaméogo G, Barro A, Taonda SJ, Hien V, 2013. La production de sorgho dans un parc à Piliostigma reticulatum en zone nordsoudanienne du Burkina Faso. Tropicultura 31 : 154-162. 